\title{
Précarité et violences: quels liens?
}

\section{Emmanuel Escard}

Département de médecine communautaire, de premier recours et des urgences, Unité interdisciplinaire de médecine et de prévention de la violence (UIMPV), Hôpitaux Universitaires de Genève (HUG)
Correspondance:

Dr Emmanuel Escard

UIMPV, Hôpitaux Universitaires de Genève (HUG)

Rue Gabrielle-Perret-Gentil 4

CH-1211 Genève 14

Tél. 0223729641

Fax 0223729645

emmanuel.escard[at]hcuge.ch
La précarité est, y compris en Suisse comme dans la plupart des pays européens, et à juste titre, parmi les principales préoccupations des citoyens. Malgré les importants efforts des politiques et ceux d'hommes ou de femmes engagés, les avancées de la lutte contre toutes les expressions de la misère restent très insuffisantes tant le phénomène est durablement ancré dans notre univers social. Caritas estime ainsi à près d'un million le nombre de personnes touchées par la pauvreté en Suisse (selon le seuil de pauvreté relatif de l'Union européenne). Nul ne semble plus à l'abri d'être un jour candidat au «toboggan social» conduisant à l'exclusion, à la détresse, à la souffrance, à la violence, et parfois à la mort, souvent par la voie du suicide. La précarité est un phénomène pluridimensionnel et dynamique par excellence, et les personnes en situation de précarité cumulent le plus souvent différents drames humains. Si la confrontation des personnes précaires à la violence n'en est pas l'aspect le plus médiatique, il n'en est pas pour autant le moindre. Non que les personnes précaires elles-mêmes, comme souhaiteraient le faire croire certains idéologistes extrémistes, en soient les principaux auteurs, mais malheureusement bien plus souvent les premières victimes.

\section{Un lien complexe et multidimensionnel}

Aucun consensus scientifique ne s'est jamais réellement imposé en ce qui concerne les liens entre précarité et violence. La précarité peut avoir diverses origines, physique, géographique, économique, psychosociale, culturelle, symbolique... Les violences peuvent intervenir dans différents contextes de vulnérabilité, comme dans un contexte multiculturel et de migration, d'environnement à risque, de limitation de l'autonomie, d'étapes particulières de vie.

Cette diversité nous empêche de considérer ou de traiter les personnes en précarité réelle ou potentielle comme une catégorie homogène et comme une population inévitablement candidate à la violence agie ou subie. Il apparaît plus judicieux de faire des distinctions entre ceux qui sont affectés par un facteur spécifique d'exclusion dans un domaine particulier (école, économie, santé, justice...), ceux qui sont victimes d'un cumul de plusieurs facteurs d'exclusion, et ceux qui ne sont pas encore exclus mais qui risquent l'exclusion si leur situation ne s'améliore pas, par exemple suite à des violences agies ou subies. Dans ces cas, la violence peut être une forme d'adaptation au milieu autant qu'une inadaptation, et elle est souvent l'expression d'un désir de vivre mieux [1].

\section{Résumé}

Les liens entre précarité et violences sont complexes et multidimensionnels. Les violences peuvent intervenir dans différents contextes de vulnérabilité, comme dans un contexte de migration, d'environnement à risque, de limitation de l'autonomie, d'étapes particulières de vie. La situation économique et sociale influe très fortement sur le degré de souffrance psychique, et le risque de violences, surtout subies ou suicidaires, peut en être augmenté. La précarité est aussi plus fréquente chez les victimes de violences intentionnelles, venant bouleverser souvent de façon durable la vie des victimes, dans des coûts qui dépassent très largement les aspects financiers.

Nous voulons rappeler la nécessité de mettre en place un dispositif complet spécialisé d'accompagnement et de suivi des personnes qui sont ou seront confrontées à des situations difficiles liées à de nombreuses pertes.

La prise en charge des conséquences des violences sur la santé doit être une mission de service public et nécessite des compétences spécifiques. L'exemple de notre activité interdisciplinaire et intégrative dans un Département hospitalier de médecine communautaire et de premier recours mériterait de se développer, notamment dans les villes de moyenne et grande taille.

La clinique de la précarité ne se fait pas hors contexte social et il est admis que la précarité traduit un affaiblissement des cadres d'intégration traditionnels [2]. Les violences impliquent des conséquences sur la santé notamment sociale, et les inégalités sociales croissantes ont des répercussions importantes sur les déterminants sociaux de la santé [3, 4]. Dix ans après la reconnaissance par l'OMS dans un Rapport mondial, des conséquences sur la santé globale des violences, problème majeur de santé 
publique, il fait l'unanimité qu'aucun facteur n'explique à lui seul pourquoi certaines personnes sont violentes envers d'autres ou plus souvent victimes de violences. Il en est de même des raisons qui font que les violences sont plus courantes dans certaines communautés que dans d'autres [5]. En effet, la violence résulte de l'interaction complexe de facteurs individuels, relationnels, communautaires et sociétaux. Il est donc fondamental de réinscrire sur un axe chronologique l'ensemble des facteurs prédisposant, contribuant et déclenchant des violences dans cette approche globale. Les facteurs de protection sont, quant à eux, les conditions qui réduisent l'impact des facteurs contribuants et prédisposants. La disponibilité des ressources dans l'entourage, des stratégies d'adaptation adéquates sont quelques-uns des facteurs qui protègent les personnes, en élargissant leur éventail d'alternatives devant des situations difficiles, voire en apparence inextricables.

\section{La précarité, source de violences}

Nous n'aborderons pas le débat sur la légitimité de la violence, ni de sa neutralisation dans le champ collectif par des processus de rationalisation idéologique faisant appel à des arguments philosophiques, mythiques, politiques, religieux, économiques... Avec la crise financière, économique et sociale qui menace l'ordre du monde aujourd'hui, les citoyens sont confrontés à la montée de la précarité. Cette situation se décline tout à la fois dans l'ordre humain (au niveau psychologique et familial) et dans l'ordre relationnel et social (école, travail, rue, etc.). La situation économique et sociale influe très fortement sur le degré de souffrance psychique. Le risque de violence et suicidaire en est alors considérablement affecté dans les cas de grande précarité. Les nouvelles formes de précarisation et d'exclusion qui traversent toutes les sphères de la vie sociale participent au développement de situations et d'états individuels et

\section{Il est fondamental de réinscrire sur un axe chronologique l'ensemble des facteurs prédisposant, contribuant et déclenchant des violences dans une approche globale.}

A certains facteurs de risque va correspondre un type particulier de violence, mais, plus généralement, les divers types de violence ont plusieurs facteurs de risque en commun. Parmi ceux-ci, nous pouvons citer la détresse psychologique, la pauvreté, la difficulté d'accès aux logements, l'isolement social, la stigmatisation de certaines populations (malades psychiatriques, sujets en situation irrégulière, anciens détenus, mères seules...). Ces problématiques sont portées par des sous-groupes sociaux qui sont dès lors considérés comme des populations «vulnérables». Au premier plan, les personnes vulnérables sont celles chez qui les principes d'autonomie, de dignité ou d'intégrité courent le risque d'être menacés ou violés.

Différents termes dans le champ médico-psychologique véhiculent des visions mécaniques et organicistes de la société. Il en est ainsi de la vulnérabilité et ses facteurs de stress et de risques, de la fragilité et ses marqueurs, de la précarité et ses bases d'incertitude et d'imprévisibilité, de l'exclusion considérée comme une réelle situation pathogène. Le succès du concept de résilience, de résistance psychique semble d'ailleurs issu des abus d'une position où ont été confondus risque et fatalité, avec des jugements péremptoires pessimistes et des pratiques nocives et excluantes [6]. C'est pourquoi, afin de ne pas réduire ces populations à leur souffrance et à leurs manques, il est important de les appréhender dans leur humanité, dans toute leur complexité, et d'insister également sur leurs ressources. collectifs combinant désordre mental et violence qui posent de nombreux problèmes à l'interprétation. L'explication du problème reste souvent prisonnière de lieux communs de la symptomatologie médicosociale. L'important devient alors de comprendre suffisamment la violence afin de savoir comment la recevoir, et de reconstruire un vécu compatible avec le désir de reconnaissance et de vivre ensemble.

Il apparaît que dans nos pays, depuis les années 1990, le vocable de détresse et de souffrance est devenu une clé de lecture des problèmes sociaux tels que la violence, les déviances et les inégalités [7, 8]. Quand le monde est bouleversé suite à des événements, ne fait plus sens, il est possible d'en être exclu. La précarité psychique va se développer d'autant plus qu'il existe une vulnérabilité psychique devant ces difficultés du monde et de la reconnaissance de soi comme digne d'existence, d'avoir une place dans sa famille, dans le groupe, dans la société. C'est une certaine mort sociale [3]. Mais faire écouter la souffrance des victimes d'inégalités sociales par le recours au «psy» ne doit pas faire oublier la nécessité d'une justice sociale.

La tentative d'établir une relation entre les processus d'exclusion et les diverses manifestations de la violence ne doit pas occulter le fait qu'il s'agit d'un problème d'équité et de respect des droits fondamentaux de tous. Cette relation devient de plus en plus dynamique et multidimensionnelle, nécessitant l'identification des différents processus qui ont abouti à l'affaiblissement voire à la rupture des liens sociaux. 


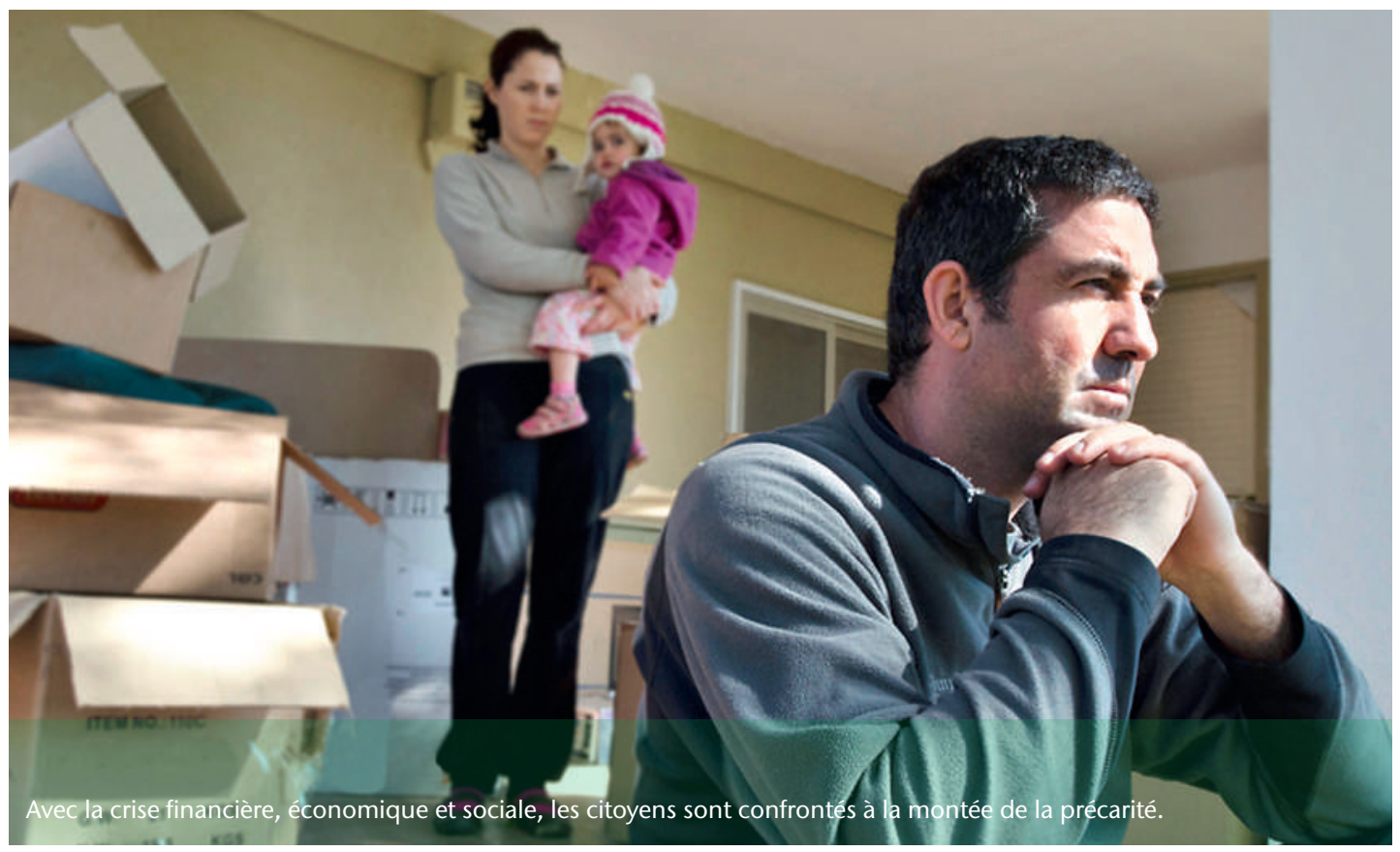

A l'instar de bien des problèmes de santé, la violence n'est pas neutre. Elle existe certes dans toutes les classes sociales, mais la recherche donne toujours à penser que les personnes de statut socio-économique inférieur y sont plus exposées. Le plus souvent, ce sont les facteurs liés à la pauvreté, plus que la pauvreté elle-même, qui accroissent le risque de violence. Les jeunes défavorisés sont ainsi plus exposés à la criminalité en tant qu'acteurs, tandis que les femmes, enfants, personnes âgées à être victimes en situation de précarité. Dans leur immense majorité, les décès suite à des violences se produisent dans des pays à faible revenu et à revenu moyen, et moins de $10 \%$ des cas dans des pays à revenu élevé (données de l'OMS). La violence est donc à la fois conséquence d'une fermeture sociale, critère de statut social et une expression d'un style de vie [9].

\section{La précarité, conséquence des violences}

Sur le plan victimologique, une étude en 2009 en France a permis de préciser que la précarité, mesurée par un score individuel EPICES (évaluation de la précarité et des inégalités de santé à partir de 11 questions), est plus fréquente chez les victimes de violences intentionnelles physiques [12]. Ceci n'apparaît pas aussi évident pour les victimes de violences psychologiques mieux réparties dans la population générale..

Les violences bouleversent souvent de façon durable la vie des victimes. Elles peuvent occasionner des années de souffrances, de mise à l'écart, de soumission, d'oubli de soi, de mise entre parenthèses des projets personnels et professionnels. L'investissement d'un cadre de suivi est difficile, souvent en pointillés. Les soins peuvent être vécus comme stig-

\section{Les médecins de premiers recours ont un rôle fondamental dans la prévention et le dépistage de la précarité et de la violence.}

Cependant, plutôt que la précarité économique, l'isolement social et moral ainsi que l'immaturité affective semblent au premier plan des situations de violence [10]. Une recherche récente en France menée par l'INSERM à propos de la maltraitance dans l'enfance a montré que cette maltraitance prend sa source beaucoup plus dans les relations interindividuelles déficientes ou carencées sur le plan psychoaffectif que dans les problèmes socio-économiques [11]. C'est dans ce contexte que certains facteurs de risque de violences vont pouvoir être déterminants (usage abusif d'alcool, schémas communautaires...). matisants, intrusifs, et la survie au quotidien est souvent prioritaire. D'où les obstacles que nous constatons à la recherche d'aide et à l'accès aux ressources auxquels les victimes doivent faire face pour se protéger et se sortir de la violence. Le sujet peut aller jusqu'à s'exclure lui-même [3]. L'isolement social n'est-il d'ailleurs pas une forme inhumaine d'oubli de celles et ceux qui souffrent en silence dans la résignation? Le délaissement est en effet un des facteurs parmi les plus fragilisants, confinant en termes de droit à la qualification pénale de vulnérabilité. Celuici est d'autant plus évident que les effets de la préca- 
rité sur la santé en limitent singulièrement les possibilités de défense ou de reconstruction psychologique, pouvant faire renoncer à demander de l'aide. Il a le plus souvent lieu dans des espaces plus ou moins grands et clos, où la misère (au sens large) se partage souvent plus facilement que la richesse, générant ainsi des zones d'exclusion et parfois de nondroit, où force publique et justice ont parfois les mêmes difficultés que la médecine à s'exprimer.

Il doit donc rester un espoir pour les auteurs et victimes de violence: celui d'être entendue en tant que personne, de retrouver l'estime de l'autre et de soi, de pouvoir réengager une communication. L'enjeu est qu'ils ne soient pas des cas stigmatisés, enkystés, désignés comme fous parce que trop en souffrance ou comme délinquants, déviants incurables... C'est pourquoi il nous apparait primordial de favoriser une prise en charge spécialisée au sein d'une équipe et d'un réseau interdisciplinaire de ces situations. En amont, les médecins de premier recours ont un rôle fondamental dans la prévention et le dépistage de la précarité et de la violence, et des actions éducatives d'ampleur doivent être menées au niveau familial et social [13].

\section{Perspectives}

Pour que la crise socio-économique mondiale ne se transforme pas en crise sanitaire, nous voulons rappeler la nécessité de mettre en place un dispositif complet d'accompagnement et de suivi des personnes qui sont ou seront confrontées à des situations difficiles liées à de nombreuses pertes. L'orientation précoce tant des victimes que des auteurs de violence vers les structures spécialisées apparaît donc incontournable. Il revient aux soignants d'être garant d'un accès à des soins respectueux de la carte du monde du patient, en travaillant aussi sur ses propres préconstruits [14]. La prise en charge des conséquences des violences sur la santé est d'abord une mission de service public et nécessite des compétences spécifiques. L'exemple de notre activité interdisciplinaire et intégrative dans un Département hospitalier de médecine communautaire et de premier recours aux Hôpitaux Universitaires de Genève mériterait de se développer, notamment dans les villes de moyenne et grande taille.

La recherche des facteurs de précarité et d'exclusion au sens large du terme, doit être systématique lors de la prise en charge d'un auteur ou d'une victime de violences. La violence par définition exclut, même quand elle est soumise à la raison. Les violences chroniques sont plus à risque d'induire un état précaire autant chez les auteurs que les victimes. La précarité fait violence et sa prise en charge ponctuelle ne doit pas faire oublier le rôle important des violences voilées, comme les violences structurelles, symboliques et culturelles.

Devant la grande hétérogénéité des populations reçues pour une problématique violente et la diver- sité dans les trajectoires qui mènent à la violence subie ou agie, il est essentiel d'insister sur la nécessité de prendre en compte la complexité et la singularité de chaque sujet et situation afin de ne pas les réduire à leurs difficultés. Le plus souvent les vulnérabilités sont très visibles et même aveuglantes, comme la violence elle-même. Ainsi, il s'agit en présence d'une personne concernée par la violence, de rechercher et d'évaluer précisément ses capacités, ressources et systèmes de soutien, qui sont souvent méconnus ou sous-estimés par les personnes elles-mêmes et par les soignants ou intervenants du réseau médico-social. La prise en charge doit intégrer un souci pour le respect de la diversité et de la marginalité, une valorisation du parcours de vie et des ressources. Elle implique un goût pour les relations de proximité et un positionnement éthique pour défendre certaines valeurs universelles.

\section{Références}

1 Tap P. Identité et exclusion. Connexions. 2005;1,83:53-78.

2 Bresson M, De Singly F. Sociologie de la précarité: domaines et approches. Paris: Armand Colin, coll. Sociologie; 2010.

3 Furtos J. Les cliniques de la précarité. Contexte social, psychopathologie et dispositifs. Masson; 2008.

4 Bodenmann P, Jackson Y, Bischoff T, Vaucher P, Diserens EA, Madrid C et al. Précarité et déterminants sociaux de la santé: quel(s) rôle(s) pour le médecin de premier recours? Rev Med Suisse. 2009;5:845-50.

5 OMS. Rapport mondial sur la violence et la santé. Genève; 2002.

6 Thomas H. Vulnérabilité, fragilité, précarité, résilience, etc. De l'usage et de la traduction de notions éponges en sciences de l'homme et de la vie. Collections Esquisses, Recueil Alexandrie; 2008.

7 Jacques P. Souffrance psychique et souffrance sociale. Pensée plurielle. 2004;8,2:21-9.

8 Masse R. Souffrance psychique et détresse existentielle: la question du sens de la détresse au défi des mesures épidémiologiques. Rev Med Suisse. 2008;4:26-9.

9 Groenemeyer A. Précarité et violence: une question de culture. In: Mucchielli L, Zauber-Man R. Crime et insécurité: un demi-siècle de bouleversements. Paris: L'Harmattan; 2006.

10 Gignon M, Jarde O, Manaouil C. «Violence et santé», autopsie d'un plan de santé publique. Santé publique. 2010, 22(6):685-91.

11 Tursz A. Facteurs liés à l'enfant, facteurs liés aux parents. Facteurs de risque de la maltraitance dans l'enfance. La Revue du praticien. 2011;61(5):658-60.

12 Sass C, Belin S, Chatain C, Moulin JJ, Debout M, Duband S. La précarité est plus fréquente chez les victimes de violences volontaires: intérêt du score EPICES. La Presse médicale. 2009;38,6:881-92.

13 Tursz A. Violences et santé: quelles actions éducatives? La Santé de l'homme. 2007, 5/6:12-4.

14 Rey H, Rinaldi Baud I. Violence et accessibilité aux soins: vulnérabilités et ressources des soignants. Médecine et Hygiène. 2000, 58(2316):1938-42. 